of the orders 4 and 8 . Hence, there are just four groups in which $H$ is necessarily non-abelian. In two of these $G / A$ is cyclic while the quotient group is non-cyclic in the other two.

The total number of the non-abelian groups of order $2^{m}$ which contain an invariant cyclic subgroup of order $2^{m-2}$, but no such subgroup of order $2^{m-1}$ is therefore fourteen. The last four were explicitly excluded from my list of these groups which do not contain an abelian subgroup of order $2^{m-1}$ including $A^{*}$ since Burnside had considered this subject. Knowing that Burnside gave the correct number of these groups I failed to observe the compensating errors. It may be added that the title of Hallet's paper as given in both reports noted above is misleading, since every possible group of order $2^{m}$ contains an invariant subgroup of order $2^{m-2}$.

STANFORD UNIVERSITY, March, 1905.

\title{
GALILEO AND THE MODERN CONCEPT OF INFINITY.
}

BY DR. EDWARD KASNER.

(Read before the American Mathematical Society, February 27, 1904.)

The definition of an infinite assemblage, as one in which a part exists which may be put into one-to-one correspondence with the whole, recognized as fundamental in recent discussions by mathematicians and philosophers, is usually associated with the names of Bolzano, Cantor, and Dedekind. The object of this note is to call attention to a passage from Galileo which is of significance in this connection.

The passage in question appears incidentally in the work which contains Galileo's most permanent contribution to science, the foundations of dynamics ; namely, the Discorsi e dimonstrazioni matematiche of $1638, \uparrow$ often referred to as the

* Transactions of the Amer. Math. Society, vol. 3 (1902), p. 385.

$\dagger$ The full title, taken from a copy of the first edition in the Columbia University library, is as follows :

Discorsi | e | Dimostrazioni | matematiche, | intorno à due nuoue scienze | Attenenti alla | Mecanica \& i Movimenti Locali, | del Signor | Galileo Galilei Linceo, | Filosofo e Matematico primario del Serenissimo|Grand Duca di Toscana. | Con vna Appendice del centro di grauità d'alcuni Solidi. | In Leida, | Appresso gli Elsevirii M.D.C. XXXVIII. 
Dialogues on motion. It is written, partly in Italian, partly in Latin, in a style comparable with that of Plato. The interlocutors are the same as those in the better known dialogues on the ptolemaic and copernican systems, published six years earlier. Salviati, in general, represents the author's own views ; Sagredo plays the rôle of the intelligent and appreciative critic ; and Simplicio is the exponent of aristotelian scholastic philosophy.

The "first day" of the Discorsi opens with a discussion of the divisibility and continuity of matter and space. This leads to a consideration of the number of points on a straight line. Simplicio remarks that under the assumption of infinite divisibility we should be forced to admit that one infinity may be larger than another, since a long segment contains more points than a short one, though both would be infinite. We now translate quite literally.*

Salv. These difficulties arise because we with our finite mind discuss the infinite, attributing to the latter properties derived from the finite and limited. This, however, is not justifiable; for the attributes great, small, and equal are not applicable to the infinite, since one cannot speak of greater, smaller, or equal infinities. An example occurs to me which I shall refer to your consideration, Signor Simplicio, since it was you who started the discussion.

I take it for granted that you know which numbers are squares and which are not.

Simpl. I am aware of the fact that a square number arises through the multiplication of any number by itself ; for example, 4 and 9 are square numbers formed from 2 and 3 .

Salv. Excellent. You remember also that just as the products are called squares, the factors, that is, the numbers which are multiplied by themselves, are called sides or roots. The remaining numbers, which are not formed from two equal factors, are called non-squares. If then I state that all numbers, squares and non-squares taken together, are more numerous than the squares taken alone, that is an obviously correct proposition, is it not?

* Page 32 in the original edition. An excellent German translation of the entire Discorsı, by von Oettingen, has appeared in Ostwald's Klassiker, Nos. 11, 24, 25; the passage mentioned hegins on page 30 of No. 11. The most recent Italian version with commentary will be found in vol. 8 of the Edizione Nazionale of Galileo's works. 
Simpl. It cannot be denied.

Salv. If now I ask how many squares are there, one can answer with truth, just as many as there are roots; for every square has a root, every root has a square, no square has more than one root, no root more than one square.

Simpl. Entirely correct.

Salv. Again, if I ask how many roots are there, one cannot deny that they are just as numerous as the complete number series, for there is no number which is not the root of some square. Admitting this, it follows that there are just as many squares as there are roots, since they are as numerous as the roots and every number is a root. Yet we said at the outset that all numbers are more numerous than all squares, since the majority of the former are non-squares. Indeed, the more numbers we take, the smaller is the proportion of squares; for up to 100 there are 10 squares, that is, one tenth are squares ; up to 10000 , one hundredth ; up to 1000000 , only one thousandth. Still up to an infinitely large number, granting we can conceive it, we were compelled to admit that there are just as many squares as numbers.

Simpl. What is to be our conclusion?

Salv. I see no escape except to say : the totality of numbers is infinite, the totality of squares is infinite, the totality of roots is infinite; the multitude of squares is not less than the multitude of numbers, neither is the latter the greater; and, finally, the attributes equal, greater and less are not applicable to infinite, but solely to finite quantities.*

The ensuing discussion is exceedingly interesting. It deals with the paradox "unity is infinity," the number of parts of a segment as distinguished from the number of points, and the straight line considered as a member of a family of circles. But enough has been cited for the present purpose.

Columbia University.

\footnotetext{
* Io non veggo che ad altra decisioni si possa venire, che à dire infiniti essere tutti $\mathrm{i}$ numeri, infiniti i quadrati, infinite le loro radici ; nè la molti tudine de' quadrati esser minore di quella di tutti i numeri, nè questa maggior di quella; \& in voltima conclusione gli attributi di eguale, maggiore, e minore non hauer luogo ne gl'infiniti, ma solo nelle quantità terminate.
} 